\title{
Lepton Flavor Violation in the singlet-triplet scotogenic model
}

\author{
Paulina Rocha-Morán ${ }^{a, b}$ and Avelino Vicente ${ }^{a}$ \\ ${ }^{a}$ Instituto de Física Corpuscular, CSIC-Universitat de València, \\ Apdo. 22085, E-46071 Valencia, Spain \\ ${ }^{b}$ Bethe Center for Theoretical Physics and Physikalisches Institut der Universität Bonn, \\ Nussallee 12, D-53115 Bonn, Germany \\ E-mail: procha@th.physik.uni-bonn.de, avelino.vicente@ific.uv.es
}

ABstRACT: We investigate lepton flavor violation (LFV) in the the singlet-triplet scotogenic model in which neutrinos acquire non-zero masses at the 1-loop level. In contrast to the most popular variant of this setup, the singlet scotogenic model, this version includes a triplet fermion as well as a triplet scalar, leading to a scenario with a richer dark matter phenomenology. Taking into account results from neutrino oscillation experiments, we explore some aspects of the LFV phenomenology of the model. In particular, we study the relative weight of the dipole operators with respect to other contributions to the LFV amplitudes and determine the most constraining observables. We show that in large portions of the parameter space, the most promising experimental perspectives are found for LFV 3-body decays and for coherent $\mu-e$ conversion in nuclei.

Keywords: Neutrino Physics, Beyond Standard Model

ARXIV EPRINT: 1605.01915 


\section{Contents}

1 Introduction 1

2 The model 2

2.1 Symmetry breaking and scalar sector 4

2.2 Neutrino masses 5

2.3 Dark matter $\quad 7$

3 LFV observables $\quad 8$

3.1 Current experimental situation and future projects 8

3.2 Approximate expressions for the observables 9

4 Phenomenological analysis $\quad 11$

5 Summary and conclusions $\quad 16$

$\begin{array}{ll}\text { A General LFV Lagrangian } & 18\end{array}$

B Generic expressions for the LFV observables 18

B.1 $\ell_{\alpha} \rightarrow \ell_{\beta} \gamma \quad 18$

$\begin{array}{lll}\text { B.2 } \ell_{\alpha} \rightarrow 3 \ell_{\beta} & 19\end{array}$

B.3 $\mu-e$ conversion in nuclei 19

\section{Introduction}

Although the Standard Model (SM) of particle physics is supported by a vast amount of experimental evidences, it is also known to be incomplete due to its lack of solution for two central problems of modern physics: neutrino masses and the dark matter (DM) of the universe. Several SM extensions aiming at a common explanation for these two issues have been put forward in recent years. The scotogenic model, proposed by Ernest Ma in [1], constitutes one of the most attractive proposals. In this model, the SM particle content is enlarged with the introduction of a second scalar doublet and $N_{N}$ (with $N_{N} \geq 2$ ) singlet fermions, all charged under a $\mathbb{Z}_{2}$ parity. This discrete symmetry forbids the usual treelevel contribution to neutrino masses, which are induced at the 1-loop level, and gives rise to a stable state, a weakly-interacting dark matter candidate. The phenomenology of this model has been studied in great detail, see [2-23], and several theoretical aspects have been discussed in the recent literature, such as renormalization group running effects [24-26] as well as new model building directions [27-32].

In this work we will concentrate on a simple extension ${ }^{1}$ of the minimal setup introduced in [1]: the singlet-triplet scotogenic model [34]. In this variant of the scotogenic model,

\footnotetext{
${ }^{1}$ See [33] for a general classification of scotogenic models leading to radiative neutrino masses and viable dark matter candidates.
} 
the fermion sector includes the $\mathrm{SU}(2)_{L}$ triplet $\Sigma$, which can mix with the singlet fermions via the vacuum expectation value $(\mathrm{VEV})$ of a real scalar, $\Omega$, also triplet under $\mathrm{SU}(2)_{L}$. The most relevant features of the minimal model, radiative neutrino masses and a stable dark matter candidate, are kept in this variant, while the singlet-triplet mixing allows one to interpolate between pure singlet DM [1] and pure triplet DM [35], when the dark matter candidate is fermionic. This leads to a richer phenomenology, in particular to better prospects in direct DM detection experiments [34].

Lepton flavor violation (LFV) is one of the most important probes of models with extended lepton sectors. In fact, precision high-intensity experiments are sensitive to the existence of new physics at very high energies, which makes flavor physics a powerful discovery tool, as demonstrated by its central role in the making of the Standard Model. Furthermore, very promising experimental projects in the search for LFV will begin their operation in the near future. In addition to the planned upgrade for the MEG experiment, which will improve its sensitivity to $\mu \rightarrow e \gamma$ branching ratios as low as $6 \cdot 10^{-14}$ [36], other new experiments will also join the effort. Among them, one can highlight the Mu3e experiment [37], which will look for the 3-body decay $\mu \rightarrow 3 e$, as well as a plethora of experiments looking for $\mu-e$ conversion in nuclei, like Mu2e [38-40], DeeMe [41], COMET [42, 43] and PRISM/PRIME [44], in all cases with spectacular sensitivity improvements compared to previous experiments. This remarkable multi-channel experimental effort in the search for LFV encourages detailed LFV studies in specific neutrino mass models.

We study LFV in the singlet-triplet scotogenic model, in the spirit of previous works for the singlet [15] and triplet [45] models. ${ }^{2}$ We will show that the model contains large regions of parameter space with observable LFV rates and hence will be probed in the near round of LFV experiments. Furthermore, we will explore some aspects of the LFV phenomenology of the model, such as the relative weight of the dipole operators with respect to other contributions to the LFV amplitudes, and determine that the most promising experimental perspectives are found for the LFV 3-body decays $\mu \rightarrow 3 e$ and for coherent $\mu-e$ conversion in nuclei.

The rest of the paper is organized as follows: in section 2 we introduce the model whereas in section 3 we review the current experimental situation in the search for LFV and obtain approximate expressions for the observables of interest. Section 4 contains our phenomenological analysis of the model. Finally, we summarize our results and draw our conclusions in section 5 and present additional analytical results in appendices A and B.

\section{The model}

We consider the singlet-triplet scotogenic model introduced in [34]. The matter content of the model, as well as the charge assignment under $\mathrm{SU}(2)_{L}, \mathrm{U}(1)_{Y}$ and $\mathbb{Z}_{2}$, is shown in table 1 . The quark sector, not included in this table, is SM-like and has $\mathbb{Z}_{2}=+1$. The new fields beyond the SM particle content include two fermions: the singlet $N$ and the triplet $\Sigma$, both with vanishing hypercharge and odd under the discrete $\mathbb{Z}_{2}$. Regarding the new

\footnotetext{
${ }^{2}$ See also [23] for a general study of LFV in scotogenic models with higher $\mathrm{SU}(2)_{L}$ representations.
} 


\begin{tabular}{|c|c|c|c|c|c|c|c|}
\hline & \multicolumn{2}{|c|}{ Standard Model } & \multicolumn{2}{|c|}{ Fermions } & \multicolumn{2}{|c|}{ Scalars } \\
\cline { 2 - 8 } & $L$ & $e$ & $\phi$ & $\Sigma$ & $N$ & $\eta$ & $\Omega$ \\
\hline generations & 3 & 3 & 1 & 1 & 1 & 1 & 1 \\
$\mathrm{SU}(2)_{L}$ & 2 & 1 & 2 & 3 & 1 & 2 & 3 \\
$\mathrm{U}(1)_{Y}$ & $-1 / 2$ & -1 & $1 / 2$ & 0 & 0 & $1 / 2$ & 0 \\
$\mathbb{Z}_{2}$ & + & + & + & - & - & - & + \\
\hline
\end{tabular}

Table 1. Matter content and charge assignment of the singlet-triplet scotogenic model.

scalars, these are the doublet $\eta$, also odd under $\mathbb{Z}_{2}$, and the real triplet $\Omega$. The $\mathrm{SU}(2)_{L}$ doublets $\phi$ and $\eta$ can be decomposed as

$$
\phi=\left(\begin{array}{c}
\phi^{+} \\
\phi^{0}
\end{array}\right), \quad \eta=\left(\begin{array}{c}
\eta^{+} \\
\eta^{0}
\end{array}\right),
$$

and can be identified with the usual SM Higgs doublet and a new inert doublet. Regarding the $\mathrm{SU}(2)_{L}$ triplets, $\Sigma$ and $\Omega$, they are decomposed using the standard $2 \times 2$ matrix notation as

$$
\Sigma=\left(\begin{array}{cc}
\frac{\Sigma^{0}}{\sqrt{2}} & \Sigma^{+} \\
\Sigma^{-} & -\frac{\Sigma^{0}}{\sqrt{2}}
\end{array}\right), \quad \Omega=\left(\begin{array}{cc}
\frac{\Omega^{0}}{\sqrt{2}} & \Omega^{+} \\
\Omega^{-} & -\frac{\Omega^{0}}{\sqrt{2}}
\end{array}\right) .
$$

With the charge assignment in table 1 , the most general $\mathrm{SU}(3)_{\mathrm{c}} \otimes \mathrm{SU}(2)_{\mathrm{L}} \otimes \mathrm{U}(1)_{\mathrm{Y}}$, Lorentz and $\mathbb{Z}_{2}$ invariant Yukawa Lagrangian is given by

$$
-\mathcal{L}_{Y}=Y_{e}^{\alpha \beta} \bar{L}_{\alpha} \phi e_{\beta}+Y_{N}^{\alpha} \bar{L}_{\alpha} \tilde{\eta} N+Y_{\Sigma}^{\alpha} \bar{L}_{\alpha} \tilde{\eta} \Sigma+Y_{\Omega} \bar{\Sigma} \Omega N+\text { h.c. }
$$

Here we indicate the flavor indices $\alpha, \beta=1,2,3$ explicitly and denote $\tilde{\eta}=i \sigma_{2} \eta^{*}$, as usual. Gauge contractions are omitted for the sake of clarity. The $\Sigma$ and $N$ fermions have Majorana mass terms,

$$
-\mathcal{L}_{M}=\frac{1}{2} M_{\Sigma} \bar{\Sigma}^{c} \Sigma+\frac{1}{2} M_{N} \bar{N}^{c} N+\text { h.c. . }
$$

Finally, the scalar potential can be written $\mathrm{as}^{3}$

$$
\begin{aligned}
\mathcal{V}= & -m_{\phi}^{2} \phi^{\dagger} \phi+m_{\eta}^{2} \eta^{\dagger} \eta+\frac{\lambda_{1}}{2}\left(\phi^{\dagger} \phi\right)^{2}+\frac{\lambda_{2}}{2}\left(\eta^{\dagger} \eta\right)^{2}+\lambda_{3}\left(\phi^{\dagger} \phi\right)\left(\eta^{\dagger} \eta\right) \\
& +\lambda_{4}\left(\phi^{\dagger} \eta\right)\left(\eta^{\dagger} \phi\right)+\frac{\lambda_{5}}{2}\left[\left(\phi^{\dagger} \eta\right)^{2}+\text { h.c. }\right]-\frac{m_{\Omega}^{2}}{2} \Omega^{\dagger} \Omega \\
& +\frac{\lambda_{1}^{\Omega}}{2}\left(\phi^{\dagger} \phi\right)\left(\Omega^{\dagger} \Omega\right)+\frac{\lambda_{2}^{\Omega}}{4}\left(\Omega^{\dagger} \Omega\right)^{2}+\frac{\lambda^{\eta}}{2}\left(\eta^{\dagger} \eta\right)\left(\Omega^{\dagger} \Omega\right) \\
& +\mu_{1} \phi^{\dagger} \Omega \phi+\mu_{2} \eta^{\dagger} \Omega \eta .
\end{aligned}
$$

\footnotetext{
${ }^{3}$ The Lagrangian in eqs. (2.3), (2.4) and (2.5) differs from the one in ref. [34] in two details: (i) some redundant terms in the scalar potential have been removed and the remaining ones have been renamed, and (ii) some couplings and mass terms have been normalized differently. The $\mathrm{SU}(2)_{L}$ triplets $\Sigma$ and $\Omega$ also have a different normalization, see eq. (2.2).
} 


\subsection{Symmetry breaking and scalar sector}

We will assume that the scalar potential in eq. (2.5) is such that

$$
\left\langle\phi^{0}\right\rangle=\frac{v_{\phi}}{\sqrt{2}}, \quad\left\langle\Omega^{0}\right\rangle=v_{\Omega}, \quad\left\langle\eta^{0}\right\rangle=0
$$

with $v_{\phi}, v_{\Omega} \neq 0$. These vacuum expectation values (VEVs) are determined by means of the minimization conditions

$$
\begin{aligned}
& t_{\phi}=-m_{\phi}^{2} v_{\phi}+\frac{1}{2} \lambda_{1} v_{\phi}^{3}+\frac{1}{2} \lambda_{1}^{\Omega} v_{\phi} v_{\Omega}^{2}-\frac{1}{\sqrt{2}} v_{\phi} v_{\Omega} \mu_{1}=0, \\
& t_{\Omega}=-m_{\Omega}^{2} v_{\Omega}+\lambda_{2}^{\Omega} v_{\Omega}^{3}+\frac{1}{2} \lambda_{1}^{\Omega} v_{\phi}^{2} v_{\Omega}-\frac{1}{2 \sqrt{2}} v_{\phi}^{2} \mu_{1}=0,
\end{aligned}
$$

where $t_{i} \equiv \frac{\partial \mathcal{V}}{\partial v_{i}}$ is the tadpole of $v_{i}$. The VEVs $v_{\phi}$ and $v_{\Omega}$ break the electroweak symmetry and induce masses for the gauge bosons,

$$
\begin{aligned}
m_{W}^{2} & =\frac{1}{4} g^{2}\left(v_{\phi}^{2}+4 v_{\Omega}^{2}\right) \\
m_{Z}^{2} & =\frac{1}{4}\left(g^{2}+g^{\prime 2}\right) v_{\phi}^{2} .
\end{aligned}
$$

We note that the triplet VEV $v_{\Omega}$ contributes to the $W$ boson mass, thus receiving constraints from electroweak precision tests. We estimate that this VEV cannot be larger than about $4.5 \mathrm{GeV}$ (at $3 \sigma)$.

The scalar spectrum of the model contains the $\mathbb{Z}_{2}$-even scalars $\phi^{0}, \Omega^{0}, \phi^{ \pm}$and $\Omega^{ \pm}$, and the $\mathbb{Z}_{2}$-odd scalars $\eta^{0}$ and $\eta^{ \pm}$. In the basis $\operatorname{Re}\left(\phi^{0}, \Omega^{0}\right)$, the mass matrix for the $\mathbb{Z}_{2}$-even neutral scalars is given by

$$
\mathcal{M}_{S}^{2}=\left(\begin{array}{cc}
-m_{\phi}^{2}+\frac{3}{2} \lambda_{1} v_{\phi}^{2}+\frac{1}{2} \lambda_{1}^{\Omega} v_{\Omega}^{2}-\frac{1}{\sqrt{2}} v_{\Omega} \mu_{1} & \lambda_{1}^{\Omega} v_{\phi} v_{\Omega}-\frac{1}{\sqrt{2}} v_{\phi} \mu_{1} \\
\lambda_{1}^{\Omega} v_{\phi} v_{\Omega}-\frac{1}{\sqrt{2}} v_{\phi} \mu_{1} & -m_{\Omega}^{2}+\frac{1}{2} \lambda_{1}^{\Omega} v_{\phi}^{2}+3 \lambda_{2}^{\Omega} v_{\Omega}^{2}
\end{array}\right)
$$

The lightest of the $S$ mass eigenstates, $S_{1} \equiv h$, can be identified with the SM Higgs boson with a mass $m_{h} \simeq 126 \mathrm{GeV}$ recently discovered at the LHC, whereas the heaviest mass eigenstate, $S_{2}$, is a new heavy Higgs boson not present in the SM. Regarding the $\mathbb{Z}_{2}$-even charged scalars, their mass matrix in the basis $\left(\phi^{ \pm}, \Omega^{ \pm}\right)$takes the form

$$
\mathcal{M}_{H^{ \pm}}^{2}=\left(\begin{array}{cc}
-m_{\phi}^{2}+\frac{1}{2} \lambda_{1} v_{\phi}^{2}+\frac{1}{2} \lambda_{1}^{\Omega} v_{\Omega}^{2}+\frac{1}{\sqrt{2}} v_{\Omega} \mu_{1}+\frac{1}{4} g^{2} v_{\phi}^{2} \xi_{W^{ \pm}} & \frac{1}{\sqrt{2}} v_{\phi} \mu_{1}-\frac{1}{2} g^{2} v_{\phi} v_{\Omega} \xi_{W^{ \pm}} \\
\frac{1}{\sqrt{2}} v_{\phi} \mu_{1}-\frac{1}{2} g^{2} v_{\phi} v_{\Omega} \xi_{W^{ \pm}} & -m_{\Omega}^{2}+\frac{1}{2} \lambda_{1}^{\Omega} v_{\phi}^{2}+\lambda_{2}^{\Omega} v_{\Omega}^{2}+g^{2} v_{\Omega}^{2} \xi_{W^{ \pm}}
\end{array}\right) .
$$

One of the $H^{ \pm}$mass eigenstates is the Goldstone boson that becomes the longitudinal component of the $W$ boson, whereas the other is a physical charged scalar. In what concerns the $\mathbb{Z}_{2}$-odd scalars $\eta^{0, \pm}$, we first express the neutral $\eta^{0}$ field in terms of its CP-even and CP-odd components as

$$
\eta^{0}=\frac{1}{\sqrt{2}}\left(\eta^{R}+i \eta^{I}\right)
$$


The conservation of the $\mathbb{Z}_{2}$ symmetry implies that the $\eta^{R, I, \pm}$ fields do not mix with the rest of scalars. Their masses are given by ${ }^{4}$

$$
\begin{aligned}
& m_{\eta^{R}}^{2}=m_{\eta}^{2}+\frac{1}{2}\left(\lambda_{3}+\lambda_{4}+\lambda_{5}\right) v_{\phi}^{2}+\frac{1}{2} \lambda^{\eta} v_{\Omega}^{2}-\frac{1}{\sqrt{2}} v_{\Omega} \mu_{2} \\
& m_{\eta^{I}}^{2}=m_{\eta}^{2}+\frac{1}{2}\left(\lambda_{3}+\lambda_{4}-\lambda_{5}\right) v_{\phi}^{2}+\frac{1}{2} \lambda^{\eta} v_{\Omega}^{2}-\frac{1}{\sqrt{2}} v_{\Omega} \mu_{2} \\
& m_{\eta^{ \pm}}^{2}=m_{\eta}^{2}+\frac{1}{2} \lambda_{3} v_{\phi}^{2}+\frac{1}{2} \lambda^{\eta} v_{\Omega}^{2}+\frac{1}{\sqrt{2}} v_{\Omega} \mu_{2} .
\end{aligned}
$$

We point out that the mass difference between the neutral $\eta$ scalars is controlled by the $\lambda_{5}$ coupling, $m_{\eta^{R}}^{2}-m_{\eta^{I}}^{2}=\lambda_{5} v_{\phi}^{2}$, and thus vanishes if $\lambda_{5}=0$. This will be relevant for the generation of neutrino masses, as shown in section 2.2.

Finally, we emphasize that the vacuum in eq. (2.6) breaks $\mathrm{SU}(2)_{L} \otimes \mathrm{U}(1)_{Y} \rightarrow \mathrm{U}(1)_{Q}$ but preserves the $\mathbb{Z}_{2}$ discrete symmetry. As we will discuss below, this gives rise to the existence of a stable neutral particle which may play the role of the dark matter of the universe.

\section{$2.2 \quad$ Neutrino masses}

Before discussing neutrino masses we must comment on the $\mathbb{Z}_{2}$-odd neutral fermions. The $\mathbb{Z}_{2}$-odd fields $\Sigma^{0}$ and $N$ get mixed by the Yukawa coupling $Y_{\Omega}$ and the non-zero VEV $v_{\Omega}$. In the basis $\left(\Sigma^{0}, N\right)$, their Majorana mass matrix takes the form

$$
\mathcal{M}_{\chi}=\left(\begin{array}{cc}
M_{\Sigma} & Y_{\Omega} v_{\Omega} \\
Y_{\Omega} v_{\Omega} & M_{N}
\end{array}\right)
$$

The mass eigenstates $\chi_{1,2}$ are determined by the $2 \times 2$ orthogonal matrix $V(\alpha)$,

$$
\left(\begin{array}{l}
\chi_{1} \\
\chi_{2}
\end{array}\right)=\left(\begin{array}{cc}
\cos \alpha & \sin \alpha \\
-\sin \alpha & \cos \alpha
\end{array}\right)\left(\begin{array}{c}
\Sigma^{0} \\
N
\end{array}\right)=V(\alpha)\left(\begin{array}{c}
\Sigma^{0} \\
N
\end{array}\right)
$$

such that

$$
\tan (2 \alpha)=\frac{2 Y_{\Omega} v_{\Omega}}{M_{\Sigma}-M_{N}}
$$

The singlet-triplet scotogenic model generates Majorana neutrino masses at the 1loop level. This is shown in figure 1, which actually includes four loop diagrams, since $\eta^{0} \equiv\left(\eta^{R}, \eta^{I}\right)$ and $\chi \equiv\left(\chi_{1}, \chi_{2}\right)$. The resulting neutrino mass matrix can be written $\operatorname{as}^{5}$

$$
\begin{aligned}
\left(\mathcal{M}_{\nu}\right)_{\alpha \beta} & =\sum_{\sigma=1}^{2}\left(\frac{i h_{\alpha \sigma}}{\sqrt{2}}\right)\left(\frac{-i h_{\beta \sigma}}{\sqrt{2}}\right)\left[I\left(M_{\chi_{\sigma}}^{2}, m_{\eta^{R}}^{2}\right)-I\left(M_{\chi_{\sigma}}^{2}, m_{\eta^{I}}^{2}\right)\right] \\
& =\sum_{\sigma=1}^{2} \frac{h_{\alpha \sigma} h_{\beta \sigma} M_{\chi_{\sigma}}}{2(4 \pi)^{2}}\left[\frac{m_{\eta^{R}}^{2} \ln \left(\frac{M_{\chi_{\sigma}}^{2}}{m_{\eta^{R}}^{2}}\right)}{M_{\chi^{2}}^{2}-m_{\eta^{R}}^{2}}-\frac{m_{\eta^{I}}^{2} \ln \left(\frac{M_{\chi_{\sigma}}^{2}}{m_{\eta^{I}}^{2}}\right)}{M_{\chi^{2}}^{2}-m_{\eta^{I}}^{2}}\right],
\end{aligned}
$$

\footnotetext{
${ }^{4}$ Although we provide analytical expressions for the masses in full generality, our analysis will assume $\mathrm{CP}$ conservation in the scalar sector, allowing us to consider that $\eta^{R}$ and $\eta^{I}$ do not mix.

${ }^{5}$ We correct this expression by including a factor of $1 / 2$ missing in [34].
} 


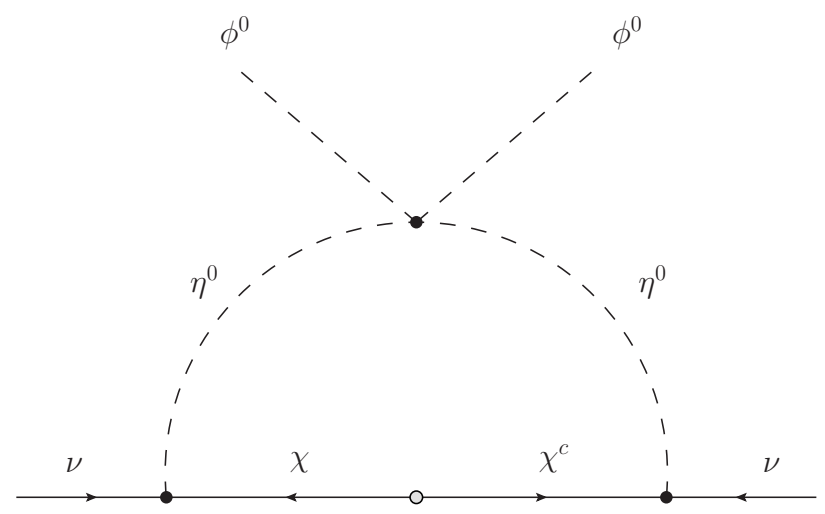

Figure 1. 1-loop neutrino masses in the singlet-triplet scotogenic model. Here $\eta^{0} \equiv\left(\eta^{R}, \eta^{I}\right)$ and $\chi \equiv\left(\chi_{1}, \chi_{2}\right)$.

where $h$ is a $3 \times 2$ matrix defined as

$$
h=\left(\begin{array}{c}
\frac{Y_{\Sigma}^{1}}{\sqrt{2}} Y_{N}^{1} \\
\frac{Y_{\Sigma}^{2}}{\sqrt{2}} Y_{N}^{2} \\
Y_{\Sigma}^{3} \\
\frac{\sqrt{2}}{\sqrt{2}} Y_{N}^{3}
\end{array}\right) \cdot V^{T}(\alpha)
$$

and $I\left(m_{1}^{2}, m_{2}^{2}\right)$ is a Passarino-Veltman function evaluated in the limit of zero external momentum. We note that $m_{\eta^{R}}^{2}=m_{\eta^{I}}^{2}$ leads to vanishing neutrino masses due to an exact cancellation between the $\eta^{R}$ and $\eta^{I}$ loops. This was indeed expected, since $m_{\eta^{R}}^{2}=m_{\eta^{I}}^{2}$ implies $\lambda_{5}=0$ and a definition of a conserved lepton number would be possible in this case. Furthermore, this justifies the choice $\lambda_{5} \ll 1$, which is natural in the sense of 't Hooft [46], given that the limit $\lambda_{5} \rightarrow 0$ increases the symmetry of the model.

It proves convenient to write the neutrino mass matrix in eq. (2.20) as

$$
\mathcal{M}_{\nu}=h \Lambda h^{T}
$$

where

$$
\Lambda=\left(\begin{array}{cc}
\Lambda_{1} & 0 \\
0 & \Lambda_{2}
\end{array}\right), \quad \Lambda_{\sigma}=\frac{M_{\chi_{\sigma}}}{2(4 \pi)^{2}}\left[\frac{m_{\eta^{R}}^{2} \ln \left(\frac{M_{\chi_{\sigma}}^{2}}{m_{\eta^{R}}^{2}}\right)}{M_{\chi_{\sigma}}^{2}-m_{\eta^{R}}^{2}}-\frac{m_{\eta^{I}}^{2} \ln \left(\frac{M_{\chi_{\sigma}}^{2}}{m_{\eta^{I}}^{2}}\right)}{M_{\chi_{\sigma}}^{2}-m_{\eta^{I}}^{2}}\right]
$$

A neutrino mass matrix as the one in eq. (2.22) formally resembles that obtained in the standard type-I seesaw with two generations of right-handed neutrinos. In this case we can make use of an adapted Casas-Ibarra parameterization [47, 48] to obtain an expression for the Yukawa matrix $h$,

$$
h=U^{*} \sqrt{\widehat{\mathcal{M}}_{\nu}} R \sqrt{\Lambda}^{-1} .
$$


Here $R$ is a $3 \times 2$ complex matrix such that $R R^{T}=\mathbb{I}_{3}$, where $\mathbb{I}_{3}$ is the $3 \times 3$ unit matrix, and the neutrino mass matrix is diagonalized as

$$
U^{T} \mathcal{M}_{\nu} U=\widehat{\mathcal{M}}_{\nu} \equiv\left(\begin{array}{ccc}
m_{1} & 0 & 0 \\
0 & m_{2} & 0 \\
0 & 0 & m_{3}
\end{array}\right)
$$

where

$$
U=\left(\begin{array}{ccc}
c_{12} c_{13} & s_{12} c_{13} & s_{13} e^{i \delta} \\
-s_{12} c_{23}-c_{12} s_{23} s_{13} e^{-i \delta} & c_{12} c_{23}-s_{12} s_{23} s_{13} e^{-i \delta} & s_{23} c_{13} \\
s_{12} s_{23}-c_{12} c_{23} s_{13} e^{-i \delta} & -c_{12} s_{23}-s_{12} c_{23} s_{13} e^{-i \delta} & c_{23} c_{13}
\end{array}\right)
$$

is the PMNS (Pontecorvo-Maki-Nakagawa-Sakata) matrix. Here $c_{i j}=\cos \theta_{i j}, s_{i j}=\sin \theta_{i j}$ and $\delta$ is the CP-violating Dirac phase. ${ }^{6}$ Similarly to the type-I seesaw with two righthanded neutrinos, the singlet-triplet scotogenic model predicts a vanishing mass for the lightest neutrino. It has, however, enough freedom to accommodate both neutrino spectra, Normal Hierarchy (NH) and Inverted Hierarchy $(\mathrm{IH})$, and the form of the complex $R$ matrix introduced in eq. (2.24) depends on this choice [48],

$$
\begin{aligned}
& R=\left(\begin{array}{cc}
0 & 0 \\
\cos \gamma & \sin \gamma \\
-\sin \gamma & \cos \gamma
\end{array}\right) \text { for } \mathrm{NH} \quad\left(m_{1}=0\right), \\
& R=\left(\begin{array}{cc}
\cos \gamma & \sin \gamma \\
-\sin \gamma & \cos \gamma \\
0 & 0
\end{array}\right) \text { for IH } \quad\left(m_{3}=0\right)
\end{aligned}
$$

We can finally make use of the previous expressions and write the Yukawa couplings $h$ in terms of the PMNS matrix $U$, the eigenvalues $m_{i}$ and the complex angle $\gamma$. In case of $\mathrm{NH}$, one obtains

$$
\begin{aligned}
& h_{\alpha 1}=\frac{1}{\sqrt{\Lambda_{1}}}\left(\cos \gamma \sqrt{m_{2}} U_{\alpha 2}^{*}-\sin \gamma \sqrt{m_{3}} U_{\alpha 3}^{*}\right), \\
& h_{\alpha 2}=\frac{1}{\sqrt{\Lambda_{2}}}\left(\sin \gamma \sqrt{m_{2}} U_{\alpha 2}^{*}+\cos \gamma \sqrt{m_{3}} U_{\alpha 3}^{*}\right),
\end{aligned}
$$

whereas for IH one finds

$$
\begin{aligned}
& h_{\alpha 1}=\frac{1}{\sqrt{\Lambda_{1}}}\left(\cos \gamma \sqrt{m_{1}} U_{\alpha 1}^{*}-\sin \gamma \sqrt{m_{2}} U_{\alpha 2}^{*}\right), \\
& h_{\alpha 2}=\frac{1}{\sqrt{\Lambda_{2}}}\left(\sin \gamma \sqrt{m_{1}} U_{\alpha 1}^{*}+\cos \gamma \sqrt{m_{2}} U_{\alpha 2}^{*}\right) .
\end{aligned}
$$

\subsection{Dark matter}

The lightest state charged under the conserved $\mathbb{Z}_{2}$ parity is stable and hence, if electrically neutral, it constitutes a standard weakly-interacting dark matter candidate. Therefore,

\footnotetext{
${ }^{6}$ In general, eq. (2.26) could also include an additional Majorana phase. However, this will not be considered in this paper.
} 
in what concerns dark matter, the singlet-triplet scotogenic model contains two distinct scenarios: (i) scalar dark matter, when the candidate is the lightest neutral $\eta$ state, $\eta_{R}$ or $\eta_{I}$, and (ii) fermion dark matter, when the candidate is $\chi_{1}$, the lightest $\chi$ state. Even though we will not be concerned about dark matter in this paper, we find it worth summarizing the main features of these two scenarios:

- Scalar dark matter. In this case the dark matter phenomenology resembles that of the inert doublet model [49] (see also [50-52] for some recent works on dark matter in the inert doublet model). Since in this scenario dark matter production in the early universe is driven by gauge interactions, there is no direct relation with LFV (driven by Yukawa interactions).

- Fermion dark matter. This scenario presents some of the most interesting features of the singlet-triplet scotogenic model [34]. The phenomenology dramatically depends on the nature of the dark matter candidate. In the two extreme cases this can be a pure $\mathrm{SU}(2)_{L}$ singlet (when $\chi_{1} \equiv N$ ) or a pure $\mathrm{SU}(2)_{L}$ triplet (when $\chi_{1} \equiv \Sigma$ ), while in general it will be an admixture of these two gauge eigenstates. When $\chi_{1}$ is mostly singlet, the dark matter phenomenology is determined by Yukawa interactions and one expects a direct link between dark matter and LFV, as in the minimal scotogenic model [20]. In contrast, the DM phenomenology of a mostly triplet dark matter candidate is driven by the known gauge interactions. This case has little impact on LFV and predicts a dark matter candidate with a mass of about $\sim 2.7 \mathrm{TeV}$ in order to reproduce the observed dark matter relic density. The parameter $Y_{\Omega}$, which determines the $N-\Sigma$ mixing, interpolates between these two cases, in a way completely analogous to DM in R-parity conserving supersymmetry.

\section{LFV observables}

\subsection{Current experimental situation and future projects}

No observation of a flavor violating process involving charged leptons has ever been made. This has been used by many experiments to set strong limits on the most relevant LFV observables, usually translated into stringent bounds on the parameter space of many new physics models. In what concerns the radiative decay $\ell_{\alpha} \rightarrow \ell_{\beta} \gamma$, the experimental search is led by the MEG collaboration. This experiment searches for the process $\mu \rightarrow e \gamma$ and recently announced the limit $\operatorname{BR}(\mu \rightarrow e \gamma)<5.7 \cdot 10^{-13}$ [53], about four times more stringent than the previous bound obtained by the same collaboration. The 3-body LFV decay $\mu \rightarrow 3 e$ was also searched for long ago by the SINDRUM experiment [54], which obtained the limit $\operatorname{BR}(\mu \rightarrow 3 e)<1.0 \cdot 10^{-12}$, still not improved by any experiment after almost 30 years. Another $\mu-e \mathrm{LFV}$ process of interest due to the existing bounds is $\mu-e$ conversion in nuclei. Among the experiments involved in this search we may mention SINDRUM II, which searched for $\mu-e$ conversion in muonic gold and obtained the impressive limit $\mathrm{CR}(\mu-e, \mathrm{Au})<7 \times 10^{-13}[55]$. Finally, the current experimental limits for $\tau$ lepton observables are less stringent, with branching ratios bounded to be below $\sim 10^{-8}$. 


\begin{tabular}{|c|c|c|}
\hline LFV Process & Present Bound & Future Sensitivity \\
\hline$\mu \rightarrow e \gamma$ & $5.7 \times 10^{-13}[53]$ & $6 \times 10^{-14}[36]$ \\
$\tau \rightarrow e \gamma$ & $3.3 \times 10^{-8}[64]$ & $\sim 3 \times 10^{-9}[59]$ \\
$\tau \rightarrow \mu \gamma$ & $4.4 \times 10^{-8}[64]$ & $\sim 3 \times 10^{-9}[59]$ \\
$\mu \rightarrow e e e$ & $1.0 \times 10^{-12}[54]$ & $\sim 10^{-16}[37]$ \\
$\tau \rightarrow \mu \mu \mu$ & $2.1 \times 10^{-8}[65]$ & $\sim 10^{-9}[59]$ \\
$\tau^{-} \rightarrow e^{-} \mu^{+} \mu^{-}$ & $2.7 \times 10^{-8}[65]$ & $\sim 10^{-9}[59]$ \\
$\tau^{-} \rightarrow \mu^{-} e^{+} e^{-}$ & $1.8 \times 10^{-8}[65]$ & $\sim 10^{-9}[59]$ \\
$\tau \rightarrow e e e$ & $2.7 \times 10^{-8}[65]$ & $\sim 10^{-9}[59]$ \\
$\mu^{-}, \mathrm{Ti} \rightarrow e^{-}, \mathrm{Ti}$ & $4.3 \times 10^{-12}[66]$ & $\sim 10^{-18}[67]$ \\
$\mu^{-}, \mathrm{Au} \rightarrow e^{-}, \mathrm{Au}$ & $7 \times 10^{-13}[55]$ & \\
$\mu^{-}, \mathrm{Al} \rightarrow e^{-}, \mathrm{Al}$ & & $10^{-15}-10^{-18}$ \\
$\mu^{-}, \mathrm{SiC} \rightarrow e^{-}, \mathrm{SiC}$ & & $10^{-14}[68]$ \\
\hline
\end{tabular}

Table 2. Current experimental bounds and future sensitivities for the most important LFV observables.

In addition to the active LFV searches, some of them with planned upgrades, several promising upcoming experiments will join the effort in the next few years. ${ }^{7}$ The MEG collaboration has announced plans for upgrades which will allow this experiment to reach a sensitivity to branching ratios as low as $6 \cdot 10^{-14}[36]$. Significant improvements are also expected for $\tau$ observables from searches in B factories [59,60], although the expected sensitivities are still less spectacular than those for $\mu$ observables. Regarding the new projects, the most promising ones are expected in searches for $\mu \rightarrow 3 e$ and $\mu-e$ conversion in nuclei. The Mu3e experiment, which plans to start data taking soon, announces a sensitivity for $\mu \rightarrow 3 e$ branching ratios of the order of $\sim 10^{-16}$ [37]. In case no discovery is made, this would imply an impressive improvement of the current bound by 4 orders of magnitude. Regarding $\mu-e$ conversion in nuclei, the competition will be shared by several experiments, with expected sensitivities for the conversion rate ranging from $10^{-14}$ to an impressive $10^{-18}$. These include Mu2e [38-40], DeeMe [41], COMET [42, 43] and, in the long term, the future PRISM/PRIME [44].

Finally, even though in this paper we concentrate on low-energy processes, we emphasize that colliders can also play a relevant role in the search for LFV. For instance, there is currently an intriguing hint at CMS for Higgs boson LFV decays into $\tau \mu$ [61]. This anomaly seems to require an explanation based on an extended scalar sector (see e.g. [62, 63]), in principle not related to the problem of neutrino masses, and cannot be accommodated in the model under investigation. For reference, in table 2 we collect present bounds and expected sensitivities for the most popular low-energy LFV observables.

\subsection{Approximate expressions for the observables}

We use the FlavorKit [69] functionality of SARAH [70-74] for the analytical computation of the LFV Wilson coefficients and observables. This allows us to automatically obtain

\footnotetext{
${ }^{7}$ See $[56-58]$ for recent reviews.
} 

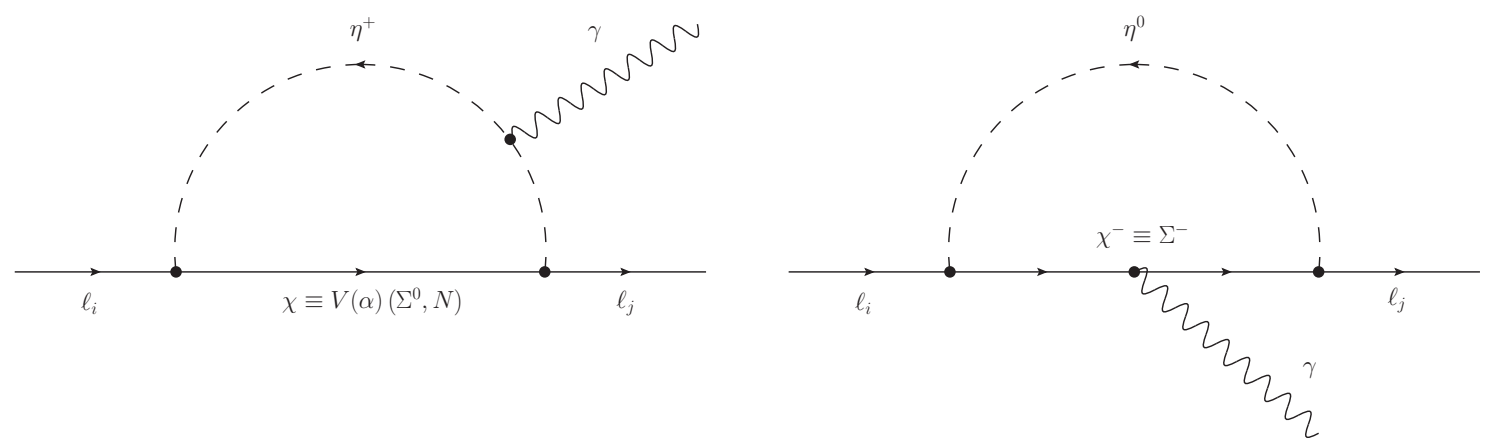

Figure 2. Photon penguin diagrams leading to the dominant Wilson coefficients $K_{1}^{L}$ and $K_{2}^{R}$.

complete analytical results for the LFV observables as well as robust numerical routines to be combined with SPheno [75, 76]. For the conventions used in this paper, the definition of the relevant LFV operators and the generic expressions for the LFV observables we refer to appendices A and B. Even though we will make use of the complete analytical results for the numerical exploration of the phenomenology of the model, we find it convenient to present simple approximate expressions for the observables of interest.

Our numerical analysis reveals that the LFV phenomenology is mainly driven by two Wilson coefficients, both generated by photon penguin diagrams: the monopole $K_{1}^{L}$ and the dipole $K_{2}^{R}$. Box diagrams also lead to sizable contributions, mainly to the Wilson coefficients $A_{L L}^{V}, B_{L L}^{V}$ and $C_{L L}^{V}$, but we have found them to be always subdominant compared to the photonic monopole and dipole contributions. Therefore, we can obtain simple approximate expressions for the LFV observables in terms of only $K_{1}^{L}$ and $K_{2}^{R}$.

The most relevant photon penguin diagrams in the singlet-triplet scotogenic model are shown in figure 2 . The diagram with the neutral fermions $\chi \equiv\left(\chi_{1}, \chi_{2}\right)$ running in the loop is common to the scotogenic model [15], whereas the diagram with the charged $\Sigma^{-}$ state is only present in the singlet-triplet variant. This difference has an impact on the phenomenology, as we will see below. Let us first consider the dipole coefficient $K_{2}^{R}$, which induces the radiative LFV decay $\ell_{\alpha} \rightarrow \ell_{\beta} \gamma$. It can be written as

$$
K_{2}^{R}=\frac{1}{16 \pi^{2}}\left(D^{0}+D^{-}\right)
$$

where the contributions from the two diagrams in figure 2 are approximately given by

$$
\begin{aligned}
D^{0} & =\frac{1}{2 m_{\eta^{+}}^{2}} \times \\
& {\left[\left(\frac{1}{\sqrt{2}} \cos \alpha \sin \alpha\left(\left(Y_{\Sigma}^{i}\right)^{*} Y_{N}^{j}+\left(Y_{N}^{i}\right)^{*} Y_{\Sigma}^{j}\right)+\frac{1}{2}\left(Y_{\Sigma}^{i}\right)^{*} Y_{\Sigma}^{j} \cos ^{2} \alpha+\left(Y_{N}^{i}\right)^{*} Y_{N}^{j} \sin ^{2} \alpha\right) F_{2}\left(\xi_{1}\right)\right.} \\
& \left.+\left(\frac{-1}{\sqrt{2}} \cos \alpha \sin \alpha\left(\left(Y_{\Sigma}^{i}\right)^{*} Y_{N}^{j}+\left(Y_{N}^{i}\right)^{*} Y_{\Sigma}^{j}\right)+\frac{1}{2}\left(Y_{\Sigma}^{i}\right)^{*} Y_{\Sigma}^{j} \sin ^{2} \alpha+\left(Y_{N}^{i}\right)^{*} Y_{N}^{j} \cos ^{2} \alpha\right) F_{2}\left(\xi_{2}\right)\right], \\
D^{-} & =-\frac{1}{2 m_{\eta^{0}}^{2}}\left(Y_{\Sigma}^{i}\right)^{*} Y_{\Sigma}^{j} G_{2}(\rho) .
\end{aligned}
$$


Similarly, the monopole coefficient $K_{1}^{L}$ can be split as

$$
K_{1}^{L}=\frac{1}{16 \pi^{2}}\left(M^{0}+M^{-}\right)
$$

and the two contributions from the penguin diagrams in figure 2 are given by

$$
\begin{aligned}
M^{0} & =-\frac{1}{6 m_{\eta^{+}}^{2}} \times \\
& \quad\left[\left(\frac{1}{\sqrt{2}} \cos \alpha \sin \alpha\left(\left(Y_{\Sigma}^{i}\right)^{*} Y_{N}^{j}+\left(Y_{N}^{i}\right)^{*} Y_{\Sigma}^{j}\right)+\frac{1}{2}\left(Y_{\Sigma}^{i}\right)^{*} Y_{\Sigma}^{j} \cos ^{2} \alpha+\left(Y_{N}^{i}\right)^{*} Y_{N}^{j} \sin ^{2} \alpha\right) F_{1}\left(\xi_{1}\right)\right. \\
& \left.+\left(\frac{-1}{\sqrt{2}} \cos \alpha \sin \alpha\left(\left(Y_{\Sigma}^{i}\right)^{*} Y_{N}^{j}+\left(Y_{N}^{i}\right)^{*} Y_{\Sigma}^{j}\right)+\frac{1}{2}\left(Y_{\Sigma}^{i}\right)^{*} Y_{\Sigma}^{j} \sin ^{2} \alpha+\left(Y_{N}^{i}\right)^{*} Y_{N}^{j} \cos ^{2} \alpha\right) F_{1}\left(\xi_{2}\right)\right],
\end{aligned}
$$

$M^{-}=\frac{1}{6 m_{\eta^{0}}^{2}}\left(Y_{\Sigma}^{i}\right)^{*} Y_{\Sigma}^{j} G_{1}(\rho)$

Here we have defined

$$
\xi_{i}=\frac{m_{\chi_{i}}^{2}}{m_{\eta^{+}}^{2}}, \quad \rho=\frac{m_{\chi^{-}}^{2}}{m_{\eta^{0}}^{2}},
$$

and used $m_{\eta^{R}}^{2} \simeq m_{\eta^{I}}^{2} \equiv m_{\eta^{0}}^{2}$. Finally, the loop functions appearing in these expressions are given by

$$
\begin{aligned}
F_{1}(x) & =\frac{2-9 x+18 x^{2}-11 x^{3}+6 x^{3} \log x}{6(1-x)^{4}}, \\
G_{1}(x) & =\frac{-16+45 x-36 x^{2}+7 x^{3}+6(3 x-2) \log x}{6(1-x)^{4}}, \\
F_{2}(x) & =\frac{1-6 x+3 x^{2}+2 x^{3}-6 x^{2} \log x}{6(1-x)^{4}} \\
G_{2}(x) & =\frac{2+3 x-6 x^{2}+x^{3}+6 x \log x}{6(1-x)^{4}}
\end{aligned}
$$

We find that in the limit $M_{\Sigma} \rightarrow \infty$ our analytical results are in good agreement with those obtained in the scotogenic model $[15] .^{8}$ Finally, we emphasize that the numerical results discussed in the next section are based on the full 1-loop evaluation of the LFV observables and not on these approximate expressions, only presented to gain insight.

\section{Phenomenological analysis}

Our phenomenological analysis uses a SARAH-generated SPheno $[75,76]$ module for the numerical evaluation of the LFV observables. We solve the tadpole equations for the squared mass terms $m_{H}^{2}$ and $m_{\Omega}^{2}$ and use an adapted Casas-Ibarra parameterization for neutrino masses to compute the Yukawa couplings $Y_{N}$ and $Y_{\Sigma}$. For this purpose, the results

\footnotetext{
${ }^{8}$ Notice that the loop functions have been renamed with respect to [15].
} 
of the global fit to neutrino oscillation data [77] will be used. Furthermore, given the little impact on the LFV phenomenology, we fix the following parameters in the scalar potential,

$$
\begin{array}{rlrl}
\lambda_{2,3,4} & =\lambda_{1,2}^{\Omega}=\lambda^{\eta}=0.1, & \lambda_{5} & =10^{-8}, \\
\mu_{1} & =50 \mathrm{GeV}, & \mu_{2}=1 \mathrm{TeV} .
\end{array}
$$

We have explicitly checked that these parameters only affect the LFV observables indirectly, due to their influence on the scalar spectrum. ${ }^{9}$ The large value chosen for the trilinear coupling $\mu_{2}$ ensures the conservation of the $\mathbb{Z}_{2}$ symmetry up to high energy scales [78]. We also fix $v_{\Omega}=1 \mathrm{GeV}$. This choice leads to a negligible deviation from $\rho=1$, thus respecting limits from electroweak precision data. Finally, the doublet VEV $v_{\phi}$ is fixed so that $m_{W}$ is correctly obtained, see eq. (2.9), and the quartic coupling $\lambda_{1}$ so that the lightest CP-even state in the model has a mass compatible with that of the recently discovered Higgs boson. This leaves us with four free model parameters,

$$
Y_{\Omega}, \quad m_{\eta}^{2}, \quad M_{N}, \quad M_{\Sigma}
$$

as well as the usual free choices in the implementation of the Casas-Ibarra parameterization: the $R$ matrix angle $\gamma$, the Dirac CP-violating phase $\delta$ and Normal/Inverted Hierarchy for the light neutrino spectrum.

General predictions of the model. We will now explore some aspects of the LFV phenomenology of the singlet-triplet scotogenic model. First of all, figure 3 shows contours of $\mathrm{BR}(\mu \rightarrow e \gamma)$ (upper left panel), $\mathrm{BR}(\mu \rightarrow 3 e)$ (upper right panel) and $\mathrm{CR}(\mu-e, \mathrm{Al})$ (lower panel) in the $m_{\eta^{-}} M_{N}$ plane, obtained with the setup introduced above and the choices $Y_{\Omega}=0.1, M_{\Sigma}=500 \mathrm{GeV}, \gamma=\delta=0$, normal hierarchy for the light neutrino spectrum and taking best-fit values for the neutrino oscillation parameters. The first conclusion one can draw from this figure is that the singlet-triplet scotogenic model will be probed in the next round of LFV experiments: one easily finds parameter points where the three observables, $\mathrm{BR}(\mu \rightarrow e \gamma), \mathrm{BR}(\mu \rightarrow 3 e)$ and $\mathrm{CR}(\mu-e, \mathrm{Al})$, are within the reach of the MEG and Mu3e experiments, respectively. In fact, the particular choice of parameters made in this figure rules out low $M_{N}$ values $(\lesssim 400 \mathrm{GeV})$ as they would imply a too large $\mu \rightarrow e \gamma$ rate, in conflict with the current bound set by the MEG experiment. In the case of $\mu \rightarrow 3 e$, the spectacular Mu3e sensitivity to branching ratios as low as $\sim 10^{-16}$ would allow one to probe the complete $m_{\eta}-M_{N}$ plane explored in figure 3 , with mass values up to the $\mathrm{TeV}$ scale and even higher in some cases. This also happens for $\mu-e$ conversion in Aluminum. In this observable, however, a strong cancellation takes place for a narrow band of the $m_{\eta}-M_{N}$ plane, where the resulting negligible conversion rates cannot be probed in the near future. Qualitatively similar results are found for $\mu-e$ conversion rates in other nuclei, where analogous cancellations take place as well.

\footnotetext{
${ }^{9}$ The parameter $\lambda_{5}$ does indeed have a strong impact on the LFV observables, but only due to the scaling of the Yukawa couplings, $Y_{N}$ and $Y_{\Sigma}$, induced via the neutrino mass relation in eq. (2.20). All our numerical results have been obtained with $\lambda_{5}=10^{-8}$, except those for the $\tau$ lepton observables, obtained with $\lambda_{5}=10^{-10}$.
} 

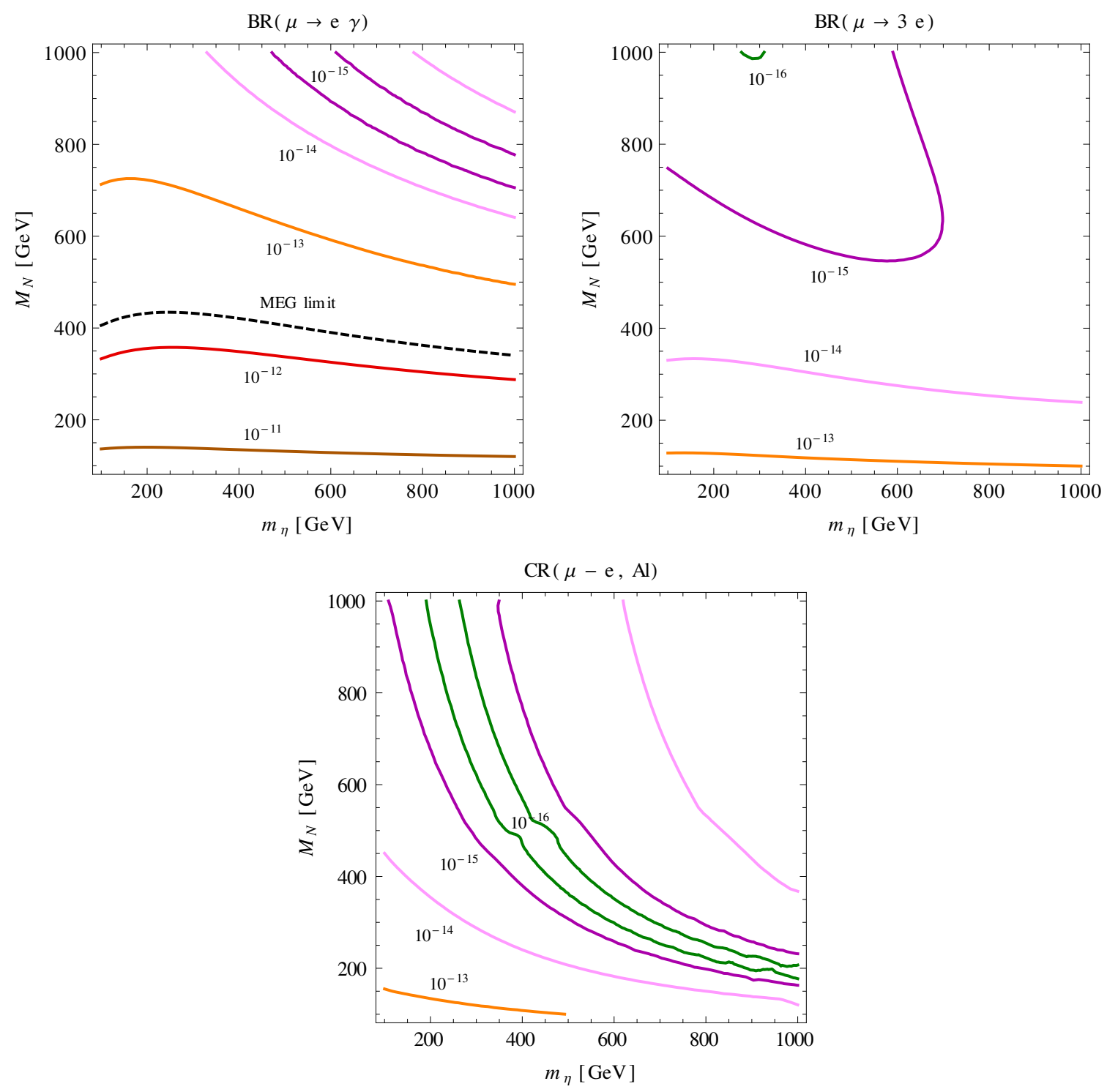

Figure 3. Contours of $\mathrm{BR}(\mu \rightarrow e \gamma), \mathrm{BR}(\mu \rightarrow 3 e)$ and $\mathrm{CR}(\mu-e, \mathrm{Al})$ in the $m_{\eta}-M_{N}$ plane. Figures obtained with fixed $Y_{\Omega}=0.1$ and $M_{\Sigma}=500 \mathrm{GeV}$, see text for more details.

Figure 3 also shows that in the long term the processes $\mu \rightarrow 3 e$ and $\mu-e$ conversion in nuclei will be more stringent than $\mu \rightarrow e \gamma$. Currently, only the MEG experiment sets relevant constraints in the explored $m_{\eta}-M_{N}$, ruling out a small portion with low $M_{N}$ values, while the current bounds for $\mu \rightarrow 3 e$ and $\mu-e$ conversion in nuclei do not imply any relevant restrictions. Given the expected experimental sensitivities in the search for these two observables, this fact will certainly change in the future. We find that the reach of experiments such as Mu3e (in case of $\mu \rightarrow 3 e$ ) and Mu2e or COMET (in case of $\mu-e$ conversion in nuclei), clearly supersedes that of MEG, even after the planned upgrade.

Before moving to the discussion of the $\mathrm{BR}(\mu \rightarrow e \gamma) / \mathrm{BR}(\mu \rightarrow 3 e)$ ratio, we would like to make some additional comments about figure 3 . We have explicitly checked that our numerical results reproduce the expected decoupling behavior, namely that all LFV 


\begin{tabular}{|c|cc|}
\hline & Point 1 & Point 2 \\
\hline$Y_{\Omega}$ & 0.1 & 0.1 \\
$m_{\eta}^{2}\left[\mathrm{GeV}^{2}\right]$ & $2.5 \cdot 10^{5}$ & $2.5 \cdot 10^{5}$ \\
$M_{N}[\mathrm{GeV}]$ & 500 & 500 \\
$M_{\Sigma}[\mathrm{GeV}]$ & 800 & 300 \\
\hline $\mathrm{BR}(\mu \rightarrow e \gamma)$ & $4.7 \cdot 10^{-13}$ & $1.3 \cdot 10^{-15}$ \\
$\mathrm{BR}(\mu \rightarrow 3 e)$ & $3.2 \cdot 10^{-15}$ & $6.1 \cdot 10^{-15}$ \\
$\mathrm{CR}(\mu-e, \mathrm{Al})$ & $1.1 \cdot 10^{-15}$ & $5.4 \cdot 10^{-14}$ \\
\hline
\end{tabular}

Table 3. Benchmark points, parameter values and LFV observables. In addition to the four input values in this table, we take the parameter choices in eqs. (4.1) and (4.2), use $\gamma=0$, best-fit values for the neutrino oscillation parameters, as obtained in [77], normal hierarchy for the light neutrino spectrum and $\delta=0$.

observables go to zero when $m_{\eta}$ and $M_{N, \Sigma}$, the masses of the particles involved in their generation, go to infinity. However, this is not completely apparent when looking at figure 3. There are two reasons for this: (i) some regions of parameter space lead to cancellations among diagrams that strongly reduce some of the Wilson coefficients (see below for details), and (ii) the fit to neutrino oscillation data that leads to an increase in the Yukawa couplings when $m_{\eta}$ or $M_{N, \Sigma}$ increase.

The $\operatorname{BR}(\mu \rightarrow 3 e) / \operatorname{BR}(\mu \rightarrow e \gamma)$ ratio. We also observe in figure 3 that for most points in the selected $m_{\eta}-M_{N}$ plane, one obtains $\mathrm{BR}(\mu \rightarrow e \gamma) \gg \mathrm{BR}(\mu \rightarrow 3 e)$. However, this is not a general prediction of the model, as we proceed to discuss now. Let us consider the benchmark points in table 3. The results for the LFV observables have been obtained making the same choices as for figure 3 , but using specific values for $m_{\eta}^{2}, M_{N}$ and $M_{\Sigma}$. First, we observe that the ratio

$$
R_{\mu e}=\frac{\operatorname{BR}(\mu \rightarrow 3 e)}{\operatorname{BR}(\mu \rightarrow e \gamma)},
$$

can vary by orders of magnitude between different benchmark points just by changing a single parameter, $M_{\Sigma}$. In fact, while point 1 predicts LFV rates within the reach of future experiments searching for $\mu \rightarrow e \gamma, \mu \rightarrow 3 e$ and $\mu-e$ conversion in nuclei, point 2 leads to a $\operatorname{BR}(\mu \rightarrow e \gamma)$ below the foreseen MEG sensitivity and can only be probed by $\mu \rightarrow 3 e$ and $\mu-e$ conversion in nuclei experiments. Moreover, we note that only $\mathrm{BR}(\mu \rightarrow e \gamma)$ varies substantially between point 1 and point 2 , with a decrease of more than two orders of magnitude, while the other $\mu-e$ flavor violating observables are slightly larger in point 2 .

The strong dependence of the $\mu \rightarrow e \gamma$ rate on $M_{\Sigma}$ can be understood as follows. When $M_{\Sigma}<M_{N}$, as in point 2, one expects the dominant LFV Feynman diagrams to be those with triplet fermions, $\Sigma^{0}$ and $\Sigma^{-}$, running in the loop. Furthermore, when the mixing between singlet and triplet fermions is small $(\alpha \simeq 0)$ one of the neutral $\chi$ states is mainly composed of $\Sigma^{0}$ and is mass degenerate with the charged $\chi^{-} \equiv \Sigma^{-}$. In this case, a cancellation between the $D^{0}$ and $D^{-}$contributions in eqs. (3.2) and (3.3) takes place. 


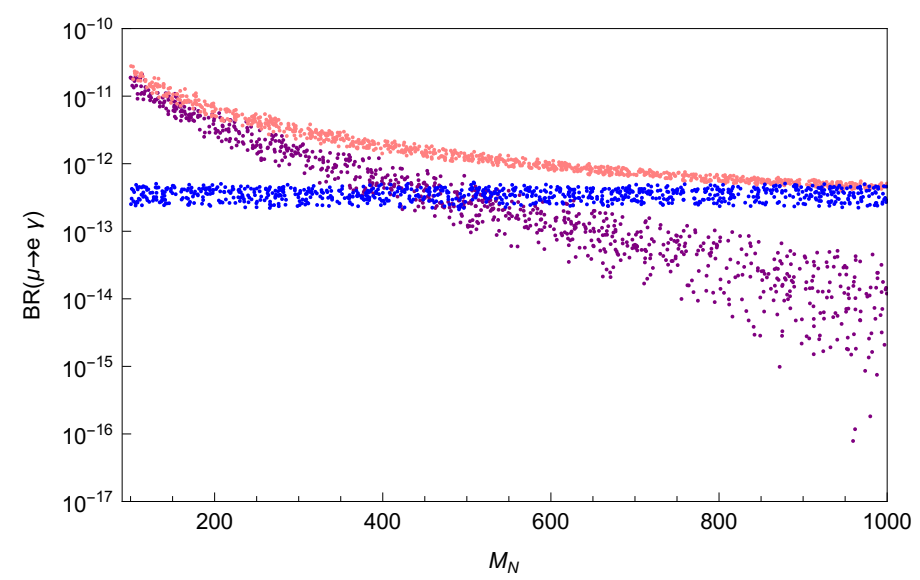

Figure 4. $\operatorname{BR}(\mu \rightarrow e \gamma)$ as a function of $M_{N}$ for fixed values $Y_{\Omega}=0.1, m_{\eta}^{2}=2.5 \cdot 10^{5} \mathrm{GeV}^{2}$ and $M_{\Sigma}=500 \mathrm{GeV}$. The purple dots display the total branching ratio, whereas the pink and blue dots show partial results obtained with only the $D^{0}$ and $D^{-}$contributions, respectively.

Using these equations, it is straightforward to show that for $\alpha \simeq 0$, the fermion triplet loops lead to $K_{2}^{R} \propto F_{2}\left(\xi_{1}\right)-2 G_{2}(\rho)$, both loop functions being positive. Therefore, one naturally expects to find parameter points where this cancellation in the dipole coefficient is effective, leading to a reduction in the $\mu \rightarrow e \gamma$ rate.

This is explicitly shown in figure 4 , where we plot our numerical results for $\mathrm{BR}(\mu \rightarrow e \gamma)$ as a function of $M_{N}$ for the fixed values $Y_{\Omega}=0.1, m_{\eta}^{2}=2.5 \cdot 10^{5} \mathrm{GeV}^{2}$ and $M_{\Sigma}=500 \mathrm{GeV}$. The purple dots display the total branching ratio, whereas the pink and blue dots show partial results obtained with only the $D^{0}$ and $D^{-}$contributions, respectively. This figure has been obtained by allowing the neutrino oscillation parameters to vary randomly within the preferred $3 \sigma$ ranges found by the global fit of [77], which explains the spread of the points. We observe that the $D^{0}$ and $D^{-}$contributions approach a common value for large $M_{N}$ values, whereas the total branching ratio drops. This is due to the abovementioned cancellation in the $\Sigma^{0}-\Sigma^{-}$loops. For low $M_{N}$ values the singlet contributions to $D^{0}$ dominate and the cancellation in the triplet contributions is not relevant. However, as $M_{N}$ increases and the $N$ contribution to $D^{0}$ gets smaller, the cancellation in the triplet contributions becomes visible. We point out that a similar cancellation in the monopole coefficient takes place, again due to the relative sign between $M^{0}$ and $M^{-}$, see eqs. (3.5) and (3.6). However, typically this cancellation has little impact on the LFV observables which receive contributions from the monopole operator due to the interplay with the other contributions (e.g. dipole).

LFV $\boldsymbol{\tau}$ decays. So far we have concentrated on $\mu-e$ violating processes. Now we turn our attention towards LFV processes involving the $\tau$ lepton. Given the worse experimental limits, these can only be phenomenologically relevant when they have rates much larger than those for the $\mu$ lepton. For example, in the benchmark points 1 and 2 presented above one finds branching ratios for the radiative decays $\tau \rightarrow \ell_{\alpha} \gamma$, with $\ell_{\alpha}=e, \mu$, in the $\sim 10^{-13}-10^{-12}$ ballpark, clearly below the expected experimental sensitivity in the near future. 

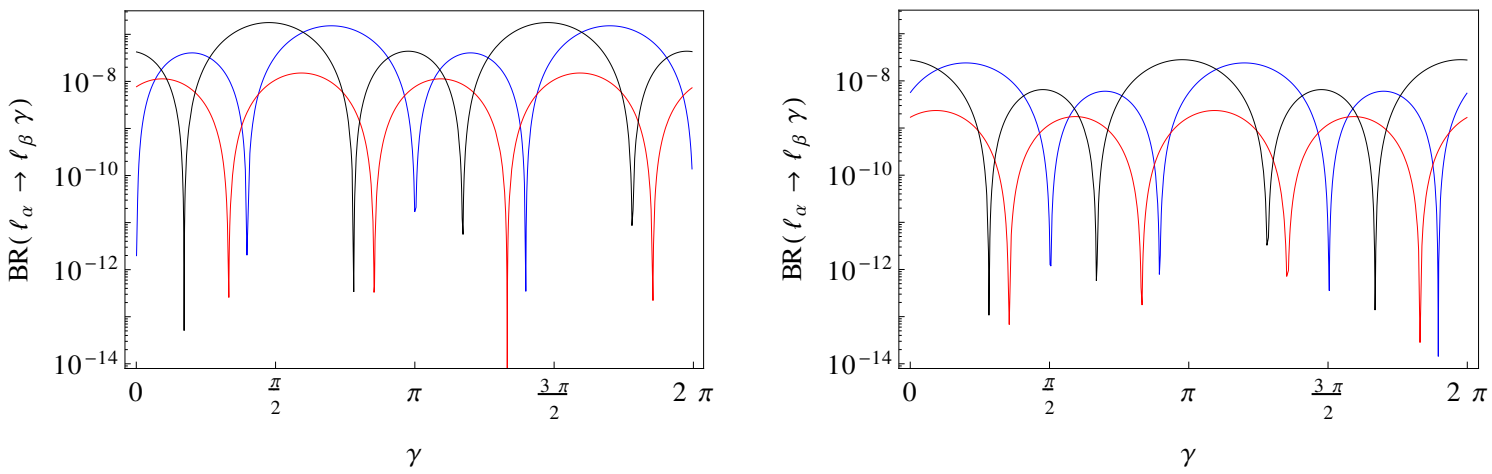

Figure 5. $\operatorname{BR}\left(\ell_{\alpha} \rightarrow \ell_{\beta} \gamma\right)$ as a function of the $R$ matrix angle $\gamma$ for $M_{\Sigma}=300 \mathrm{GeV}$ (left) and $M_{\Sigma}=800 \mathrm{GeV}$ (right). The color code is as follows: $(\alpha, \beta)=(2,1)$ in blue, $(\alpha, \beta)=(3,1)$ in red and $(\alpha, \beta)=(3,2)$ in black. See text for more details.

The results shown in table 3 for points 1 and 2 were obtained with a vanishing $R$ matrix angle $\gamma$. This parameter has a direct impact on the Yukawa couplings $Y_{N}$ and $Y_{\Sigma}$, see eqs. (2.29)-(2.32), and can lead to cancellations in the amplitudes of specific flavor violating transitions. This is illustrated in figure 5 , where we show our numerical results for $\operatorname{BR}\left(\ell_{\alpha} \rightarrow \ell_{\beta} \gamma\right)$ as a function of the $R$ matrix angle $\gamma$ (assumed to be real for simplicity) for $M_{\Sigma}=300 \mathrm{GeV}$ (on the left) and $M_{\Sigma}=800 \mathrm{GeV}$ (on the right). The rest of the parameters are fixed to the same values as in points 1 and 2 , with the exception of a smaller $\lambda_{5}$ coupling $\left(\lambda_{5}=10^{-10}\right)$ in order to increase the resulting Yukawa couplings and get larger LFV rates. We see in these figures that even though most points are experimentally excluded due to a $\mu \rightarrow e \gamma$ rate above the MEG bound, for certain $\gamma$ values a strong cancellation takes place, leading to a tiny $\mathrm{BR}(\mu \rightarrow e \gamma)$ and $\mathrm{BR}(\tau \rightarrow e \gamma) \sim 10^{-9}-10^{-8}$ within reach of $\mathrm{B}$ factories.

Therefore, we conclude that the singlet-triplet scotogenic model can also be probed via $\tau$ observables. However, the scenarios that would be experimentally explored in this way are not generic and require a certain level of tuning in the Yukawa parameters in order to suppress the $\mu \rightarrow e$ rates.

\section{Summary and conclusions}

We have investigated the lepton flavor violating phenomenology of the singlet-triplet scotogenic model, a well-motivated scotogenic neutrino mass model in which neutrinos acquire their masses at the 1-loop level. The same symmetry that forbids the tree-level generation of neutrino masses stabilizes a weakly-interacting dark matter candidate, thus providing a natural solution for another fundamental problem of current physics.

Our main findings can be summarized as follows:

- The model will be probed in the next generation of LFV experiments. In fact, we have found that parts of the parameter space are already ruled out by $\mu \rightarrow e \gamma$ searches. This of course depends on the value of the $\lambda_{5}$ parameter, which sets the global size of the Yukawa parameters and is expected to be naturally small due to its crucial role in the violation of lepton number. 
- Currently, the most stringent LFV bound on the model is the one set by the MEG experiment on $\operatorname{BR}(\mu \rightarrow e \gamma)$. However, this will soon change due to the impressive expected sensitivity in the incoming experiments. Experiments such as Mu3e (searching for $\mu \rightarrow 3 e$ ) and Mu2e or COMET (searching for $\mu-e$ conversion in nuclei) will soon probe larger portions of the parameter space of the model.

- The operators with the largest contributions to the LFV amplitudes are the monopole and dipole ones. These are induced by photon penguin diagrams with scotogenic states running in the loop. Box diagrams have a subdominant role.

- One naturally finds points of the parameter space with $\operatorname{BR}(\mu \rightarrow 3 e), \operatorname{CR}(\mu-e$, Nucleus $) \gg \operatorname{BR}(\mu \rightarrow e \gamma)$. This is caused by cancellations in the dipole coefficient which take place when the dominant contributions are generated by $\Sigma^{0}-\Sigma^{-}$loops. When this happens, MEG is usually unable to constrain the model.

- The singlet-triplet scotogenic model can also be probed via $\tau$ observables, but the scenarios where these have values close to the current or near future sensitivities require a certain tuning of the Yukawa parameters. Nevertheless, this can be achieved by properly choosing the $\gamma$ angle of the Casas-Ibarra matrix $R$.

Finally, there are other ways to probe the parameter space of the singlet-triplet scotogenic model. As already explained, scotogenic models have a potential interplay between DM physics and LFV in scenarios with fermionic DM. In this case, the application of LFV bounds combined with the Planck result for the DM relic density and contraints from direct DM detection experiments (an attractive feature of the singlet-triplet scotogenic model), would help obtaining very stringent constraints on the model and, eventually, ruling out large fractions of the parameter space. Regarding collider phenomenology, the $\Sigma$ and $\Omega$ triplets can be pair-produced in Drell-Yan processes at the LHC. In case of the $\Sigma$ fermions, their subsequent decays lead to final states including DM particles, hence to signatures with missing energy, in a way analogous to the standard R-parity conserving supersimmetric signals [79]. These interesting possibilities are left for future work.

\section{Acknowledgments}

The authors are grateful to J.W.F. Valle and R. Lineros for fruitful discussions. AV is also grateful to A. Merle, M. Platscher and N. Rojas for discussions about the singlettriplet scotogenic model and collaboration in related projects. Work supported by the Spanish grants FPA2014-58183-P, Multidark CSD2009-00064, SEV-2014-0398 (MINECO) and PROMETEOII/2014/084 (Generalitat Valenciana). PR was founded by CONACYT becas en el extranjero CVU 468534. AV acknowledges financial support from the "Juan de la Cierva" program (27-13-463B- 731) funded by the Spanish MINECO. 


\section{A General LFV Lagrangian}

The general LFV Lagrangian can be split into different pieces as ${ }^{10}$

$$
\mathcal{L}_{\mathrm{LFV}}=\mathcal{L}_{\ell \ell \gamma}+\mathcal{L}_{4 \ell}+\mathcal{L}_{2 \ell 2 q}
$$

The first term is the $\ell-\ell-\gamma$ interaction Lagrangian, generally given by

$$
\mathcal{L}_{\ell \ell \gamma}=e \bar{\ell}_{\beta}\left[\gamma^{\mu}\left(K_{1}^{L} P_{L}+K_{1}^{R} P_{R}\right)+i m_{\ell_{\alpha}} \sigma^{\mu \nu} q_{\nu}\left(K_{2}^{L} P_{L}+K_{2}^{R} P_{R}\right)\right] \ell_{\alpha} A_{\mu}+\text { h.c. }
$$

Here $e$ is the electric charge, $q$ is the photon momentum, $P_{L, R}=\frac{1}{2}\left(1 \mp \gamma_{5}\right)$ are the usual chirality projectors and the lepton flavors are denoted by $\ell_{\alpha, \beta}$. We omit flavor indices in the Wilson coefficients for the sake of clarity. The first and second terms in eq. (A.2) are usually called monopole and dipole operators, respectively. Notice that we have singled out the photonic contributions, not included in other vector operators. On the contrary, $Z$ - and Higgs boson contributions have been included whenever possible.

The most general 4-lepton interaction Lagrangian compatible with Lorentz invariance can be written as

$$
\mathcal{L}_{4 \ell}=\sum_{\substack{I=S, V, T \\ X, Y=L, R}} A_{X Y}^{I} \bar{\ell}_{\beta} \Gamma_{I} P_{X} \ell_{\alpha} \bar{\ell}_{\delta} \Gamma_{I} P_{Y} \ell_{\gamma}+\text { h.c. },
$$

where we have defined $\Gamma_{S}=1, \Gamma_{V}=\gamma_{\mu}$ and $\Gamma_{T}=\sigma_{\mu \nu}$ and $\ell_{\alpha, \beta, \gamma, \delta}$ denote the lepton flavors. Finally, the last piece of eq. (A.1) is the general $2 \ell 2 q 4$-fermion interaction Lagrangian, given by

$$
\mathcal{L}_{2 \ell 2 q}=\mathcal{L}_{2 \ell 2 d}+\mathcal{L}_{2 \ell 2 u}
$$

where

$$
\begin{aligned}
\mathcal{L}_{2 \ell 2 d} & =\sum_{\substack{I=S, V, T \\
X, Y=L, R}} B_{X Y}^{I} \bar{\ell}_{\beta} \Gamma_{I} P_{X} \ell_{\alpha} \bar{d}_{\gamma} \Gamma_{I} P_{Y} d_{\gamma}+\text { h.c. } \\
\mathcal{L}_{2 \ell 2 u} & =\left.\mathcal{L}_{2 \ell 2 d}\right|_{d \rightarrow u, B \rightarrow C}
\end{aligned}
$$

and we have used $d_{\gamma}$ to denote the d-quark flavor.

\section{B Generic expressions for the LFV observables}

\section{B.1 $\ell_{\alpha} \rightarrow \ell_{\beta} \gamma$}

The radiative decays $\ell_{\alpha} \rightarrow \ell_{\beta} \gamma$ only receive contributions from the dipole operators. The decay width is given by [80]

$$
\Gamma\left(\ell_{\alpha} \rightarrow \ell_{\beta} \gamma\right)=\frac{\alpha m_{\ell_{\alpha}}^{5}}{4}\left(\left|K_{2}^{L}\right|^{2}+\left|K_{2}^{R}\right|^{2}\right)
$$

where $\alpha$ is the electromagnetic fine structure constant.

\footnotetext{
${ }^{10}$ We closely follow the notation and conventions used in FlavorKit, see [69].
} 


\section{B.2 $\ell_{\alpha} \rightarrow 3 \ell_{\beta}$}

In this case, in addition to the standard dipole contributions, the decay width receives contributions from the monopole operators in eq. (A.2) and from the 4-lepton operators in eq. (A.3). The resulting decay width can be written as [69]

$$
\begin{aligned}
\Gamma\left(\ell_{\alpha} \rightarrow 3 \ell_{\beta}\right)= & \frac{m_{\ell_{\alpha}}^{5}}{512 \pi^{3}}\left[e^{4}\left(\left|K_{2}^{L}\right|^{2}+\left|K_{2}^{R}\right|^{2}\right)\left(\frac{16}{3} \log \frac{m_{\ell_{\alpha}}}{m_{\ell_{\beta}}}-\frac{22}{3}\right)\right. \\
& +\frac{1}{24}\left(\left|A_{L L}^{S}\right|^{2}+\left|A_{R R}^{S}\right|^{2}\right)+\frac{1}{12}\left(\left|A_{L R}^{S}\right|^{2}+\left|A_{R L}^{S}\right|^{2}\right) \\
& +\frac{2}{3}\left(\left|\hat{A}_{L L}^{V}\right|^{2}+\left|\hat{A}_{R R}^{V}\right|^{2}\right)+\frac{1}{3}\left(\left|\hat{A}_{L R}^{V}\right|^{2}+\left|\hat{A}_{R L}^{V}\right|^{2}\right)+6\left(\left|A_{L L}^{T}\right|^{2}+\left|A_{R T}^{T}\right|^{2}\right) \\
& +\frac{e^{2}}{3}\left(K_{2}^{L} A_{R L}^{S *}+K_{2}^{R} A_{L R}^{S *}+\text { c.c. }\right)-\frac{2 e^{2}}{3}\left(K_{2}^{L} \hat{A}_{R L}^{V *}+K_{2}^{R} \hat{A}_{L R}^{V *}+\text { c.c. }\right) \\
& -\frac{4 e^{2}}{3}\left(K_{2}^{L} \hat{A}_{R R}^{V *}+K_{2}^{R} \hat{A}_{L L}^{V *}+\text { c.c. }\right) \\
& \left.-\frac{1}{2}\left(A_{L L}^{S} A_{L L}^{T *}+A_{R R}^{S} A_{R R}^{T *}+\text { c.c. }\right)-\frac{1}{6}\left(A_{L R}^{S} \hat{A}_{L R}^{V *}+A_{R L}^{S} \hat{A}_{R L}^{V *}+\text { c.c. }\right)\right] .
\end{aligned}
$$

This expression combines the contributions from monopole operators with those of 4-lepton operators of vectorial type,

$$
\hat{A}_{X Y}^{V}=A_{X Y}^{V}+e^{2} K_{1}^{X} \quad(X, Y=L, R),
$$

and neglects the mass of the leptons in the final state, with the exception of the dipole contributions $K_{2}^{L, R}$, where an infrared divergence would otherwise occur due to the presence of a massless photon propagator.

\section{B.3 $\mu-e$ conversion in nuclei}

In coherent $\mu-e$ conversion in nuclei, only the scalar and vector operators in eqs. (A.2), (A.5) and (A.6) contribute. This includes photonic monopole and dipole operators, supplemented with the standard photon vertices with the up- and down quarks, as well as $2 \ell 2 q 4$-fermion operators. They induce the effective $\mu e q q$ couplings

$$
\begin{aligned}
& g_{L V(q)}=\frac{\sqrt{2}}{G_{F}}\left[e^{2} Q_{q}\left(K_{1}^{L}-K_{2}^{R}\right)-\frac{1}{2}\left(C_{\ell \ell q q}^{V L L}+C_{\ell \ell q q}^{V L R}\right)\right], \\
& g_{R V(q)}=\left.g_{L V(q)}\right|_{L \rightarrow R}, \\
& g_{L S(q)}=-\frac{\sqrt{2}}{G_{F}} \frac{1}{2}\left(C_{\ell \ell q q}^{S L L}+C_{\ell \ell q q}^{S L R}\right), \\
& g_{R S(q)}=\left.g_{L S(q)}\right|_{L \rightarrow R},
\end{aligned}
$$

where $Q_{q}$ is the quark electric charge $\left(Q_{d}=-1 / 3, Q_{u}=2 / 3\right)$ and $C_{\ell \ell q q}^{I X K}=B_{X Y}^{K}\left(C_{X Y}^{K}\right)$ for d-quarks (u-quarks), with $X=L, R$ and $K=S, V$. These couplings at the quark level 
must be dressed to obtain the effective couplings at the nucleon level. One finds

$$
\begin{aligned}
& g_{X K}^{(0)}=\frac{1}{2} \sum_{q=u, d, s}\left(g_{X K(q)} G_{K}^{(q, p)}+g_{X K(q)} G_{K}^{(q, n)}\right), \\
& g_{X K}^{(1)}=\frac{1}{2} \sum_{q=u, d, s}\left(g_{X K(q)} G_{K}^{(q, p)}-g_{X K(q)} G_{K}^{(q, n)}\right),
\end{aligned}
$$

where the $G_{K}^{(q, p)}$ and $G_{K}^{(q, n)}$ numerical coefficients were computed in [81] and given in [69]. For an improved calculation of the scalar coefficients we refer to [82]. Finally, the conversion rate, normalized to the standard muon capture rate $\Gamma_{\text {capt }}$, is given by [83]

$$
\begin{aligned}
\operatorname{CR}(\mu-e, \text { Nucleus })= & \frac{p_{e} E_{e} m_{\mu}^{3} G_{F}^{2} \alpha^{3} Z_{\mathrm{eff}}^{4} F_{p}^{2}}{8 \pi^{2} Z \Gamma_{\mathrm{capt}}} \\
& \times\left\{\left|(Z+N)\left(g_{L V}^{(0)}+g_{L S}^{(0)}\right)+(Z-N)\left(g_{L V}^{(1)}+g_{L S}^{(1)}\right)\right|^{2}+\right. \\
& \left.\left|(Z+N)\left(g_{R V}^{(0)}+g_{R S}^{(0)}\right)+(Z-N)\left(g_{R V}^{(1)}+g_{R S}^{(1)}\right)\right|^{2}\right\} .
\end{aligned}
$$

$Z$ and $N$ are the number of protons and neutrons in the nucleus under consideration and $Z_{\text {eff }}$ is its effective atomic charge [84]. Furthermore, $G_{F}$ is the Fermi constant, $F_{p}$ is the nuclear matrix element and $p_{e}$ and $E_{e}\left(\simeq m_{\mu}\right)$ are the momentum and energy of the electron.

Open Access. This article is distributed under the terms of the Creative Commons Attribution License (CC-BY 4.0), which permits any use, distribution and reproduction in any medium, provided the original author(s) and source are credited.

\section{References}

[1] E. Ma, Verifiable radiative seesaw mechanism of neutrino mass and dark matter, Phys. Rev. D 73 (2006) 077301 [hep-ph/0601225] [INSPIRE].

[2] E. Ma, Common origin of neutrino mass, dark matter and baryogenesis, Mod. Phys. Lett. A 21 (2006) 1777 [hep-ph/0605180] [INSPIRE].

[3] J. Kubo, E. Ma and D. Suematsu, Cold Dark Matter, Radiative Neutrino Mass, $\mu \rightarrow$ e $\gamma$ and Neutrinoless Double Beta Decay, Phys. Lett. B 642 (2006) 18 [hep-ph/0604114] [InSPIRE].

[4] T. Hambye, K. Kannike, E. Ma and M. Raidal, Emanations of Dark Matter: Muon Anomalous Magnetic Moment, Radiative Neutrino Mass and Novel Leptogenesis at the TeV Scale, Phys. Rev. D 75 (2007) 095003 [hep-ph/0609228] [INSPIRE].

[5] D. Aristizabal Sierra, J. Kubo, D. Restrepo, D. Suematsu and O. Zapata, Radiative seesaw: Warm dark matter, collider and lepton flavour violating signals, Phys. Rev. D 79 (2009) 013011 [arXiv:0808.3340] [INSPIRE].

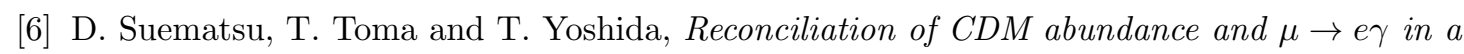
radiative seesaw model, Phys. Rev. D 79 (2009) 093004 [arXiv:0903.0287] [INSPIRE].

[7] G.B. Gelmini, E. Osoba and S. Palomares-Ruiz, Inert-Sterile Neutrino: Cold or Warm Dark Matter Candidate, Phys. Rev. D 81 (2010) 063529 [arXiv:0912.2478] [INSPIRE]. 
[8] A. Adulpravitchai, M. Lindner and A. Merle, Confronting Flavour Symmetries and extended Scalar Sectors with Lepton Flavour Violation Bounds, Phys. Rev. D 80 (2009) 055031 [arXiv: 0907.2147] [INSPIRE].

[9] M. Aoki and S. Kanemura, Probing the Majorana nature of TeV-scale radiative seesaw models at collider experiments, Phys. Lett. B 689 (2010) 28 [arXiv:1001.0092] [InSPIRE].

[10] Y.H. Ahn and H. Okada, Non-zero $\theta_{13}$ linking to Dark Matter from Non-Abelian Discrete Flavor Model in Radiative Seesaw, Phys. Rev. D 85 (2012) 073010 [arXiv:1201.4436] [INSPIRE].

[11] D. Schmidt, T. Schwetz and T. Toma, Direct Detection of Leptophilic Dark Matter in a Model with Radiative Neutrino Masses, Phys. Rev. D 85 (2012) 073009 [arXiv:1201.0906] [INSPIRE].

[12] E. Ma, Radiative Scaling Neutrino Mass and Warm Dark Matter, Phys. Lett. B 717 (2012) 235 [arXiv: 1206.1812] [INSPIRE].

[13] S. Kashiwase and D. Suematsu, Baryon number asymmetry and dark matter in the neutrino mass model with an inert doublet, Phys. Rev. D 86 (2012) 053001 [arXiv:1207.2594] [INSPIRE].

[14] S. Kashiwase and D. Suematsu, Leptogenesis and dark matter detection in a TeV scale neutrino mass model with inverted mass hierarchy, Eur. Phys. J. C 73 (2013) 2484 [arXiv: 1301.2087] [INSPIRE].

[15] T. Toma and A. Vicente, Lepton Flavor Violation in the Scotogenic Model, JHEP 01 (2014) 160 [arXiv: 1312.2840] [INSPIRE].

[16] J. Racker, Mass bounds for baryogenesis from particle decays and the inert doublet model, JCAP 03 (2014) 025 [arXiv: 1308.1840] [INSPIRE].

[17] M. Klasen, C.E. Yaguna, J.D. Ruiz-Alvarez, D. Restrepo and O. Zapata, Scalar dark matter and fermion coannihilations in the radiative seesaw model, JCAP 04 (2013) 044 [arXiv: 1302.5298] [INSPIRE].

[18] S.-Y. Ho and J. Tandean, Probing Scotogenic Effects in Higgs Boson Decays, Phys. Rev. D 87 (2013) 095015 [arXiv: 1303.5700] [InSPIRE].

[19] S.-Y. Ho and J. Tandean, Probing Scotogenic Effects in $e^{+} e^{-}$Colliders, Phys. Rev. D 89 (2014) 114025 [arXiv: 1312.0931] [INSPIRE].

[20] A. Vicente and C.E. Yaguna, Probing the scotogenic model with lepton flavor violating processes, JHEP 02 (2015) 144 [arXiv:1412.2545] [INSPIRE].

[21] G. Faisel, S.-Y. Ho and J. Tandean, Exploring X-Ray Lines as Scotogenic Signals, Phys. Lett. B 738 (2014) 380 [arXiv: 1408.5887] [INSPIRE].

[22] E. Molinaro, C.E. Yaguna and O. Zapata, FIMP realization of the scotogenic model, JCAP 07 (2014) 015 [arXiv: 1405.1259] [INSPIRE].

[23] T.A. Chowdhury and S. Nasri, Lepton Flavor Violation in the Inert Scalar Model with Higher Representations, JHEP 12 (2015) 040 [arXiv: 1506.00261] [INSPIRE].

[24] R. Bouchand and A. Merle, Running of Radiative Neutrino Masses: The Scotogenic Model, JHEP 07 (2012) 084 [arXiv: 1205.0008] [INSPIRE].

[25] A. Merle and M. Platscher, Parity Problem of the Scotogenic Neutrino Model, Phys. Rev. D 92 (2015) 095002 [arXiv: 1502.03098] [INSPIRE]. 
[26] A. Merle and M. Platscher, Running of radiative neutrino masses: the scotogenic model revisited, JHEP 11 (2015) 148 [arXiv:1507.06314] [INSPIRE].

[27] E. Ma, Dark Scalar Doublets and Neutrino Tribimaximal Mixing from $A_{4}$ Symmetry, Phys. Lett. B 671 (2009) 366 [arXiv:0808.1729] [INSPIRE].

[28] A. Adulpravitchai, M. Lindner, A. Merle and R.N. Mohapatra, Radiative Transmission of Lepton Flavor Hierarchies, Phys. Lett. B 680 (2009) 476 [arXiv:0908. 0470] [INSPIRE].

[29] E. Ma, I. Picek and B. Radovčić, New Scotogenic Model of Neutrino Mass with U(1) $)_{D}$ Gauge Interaction, Phys. Lett. B 726 (2013) 744 [arXiv:1308.5313] [INSPIRE].

[30] E. Ma and A. Natale, Scotogenic $Z_{2}$ or $\mathrm{U}(1)_{D}$ Model of Neutrino Mass with $\Delta(27)$ Symmetry, Phys. Lett. B 734 (2014) 403 [arXiv:1403.6772] [INSPIRE].

[31] J.-H. Yu, Hidden Gauged U(1) Model: Unifying Scotogenic Neutrino and Flavor Dark Matter, Phys. Rev. D 93 (2016) 113007 [arXiv:1601.02609] [INSPIRE].

[32] A. Ahriche, K.L. McDonald and S. Nasri, The Scale-Invariant Scotogenic Model, JHEP 06 (2016) 182 [arXiv: 1604.05569] [INSPIRE].

[33] D. Restrepo, O. Zapata and C.E. Yaguna, Models with radiative neutrino masses and viable dark matter candidates, JHEP 11 (2013) 011 [arXiv:1308.3655] [INSPIRE].

[34] M. Hirsch, R.A. Lineros, S. Morisi, J. Palacio, N. Rojas and J.W.F. Valle, WIMP dark matter as radiative neutrino mass messenger, JHEP 10 (2013) 149 [arXiv:1307.8134] [INSPIRE].

[35] E. Ma and D. Suematsu, Fermion Triplet Dark Matter and Radiative Neutrino Mass, Mod. Phys. Lett. A 24 (2009) 583 [arXiv:0809.0942] [INSPIRE].

[36] A.M. Baldini et al., MEG Upgrade Proposal, arXiv:1301.7225 [InSPIRE].

[37] A. Blondel et al., Research Proposal for an Experiment to Search for the Decay $\mu \rightarrow$ eee, arXiv: 1301.6113 [INSPIRE].

[38] Mu2E collaboration, R.M. Carey et al., Proposal to search for $\mu^{-} N \rightarrow e^{-} N$ with a single event sensitivity below $10^{-16}$, FERMILAB-PROPOSAL-0973 [INSPIRE].

[39] Mu2E collaboration, D. Glenzinski, The Mu2e Experiment at Fermilab, AIP Conf. Proc. 1222 (2010) 383 [INSPIRE].

[40] Mu2E collaboration, R.J. Abrams et al., Mu2e Conceptual Design Report, arXiv:1211.7019 [INSPIRE].

[41] M. Aoki, A New Idea for an Experimental Search for $\mu$-e Conversion, PoS (ICHEP 2010) 279.

[42] COMET collaboration, Y.G. Cui et al., Conceptual design report for experimental search for lepton flavor violating $\mu^{-}-e^{-}$conversion at sensitivity of $10^{-16}$ with a slow-extracted bunched proton beam (COMET), KEK-2009-10 [INSPIRE].

[43] Y. Kuno, A search for muon-to-electron conversion at J-PARC: the COMET experiment, Prog. Theor. Exp. Phys. 2013 (2013) 022C01.

[44] R.J. Barlow, The PRISM/PRIME project, Nucl. Phys. Proc. Suppl. 218 (2011) 44 [inSPIRE].

[45] W. Chao, Dark matter, LFV and neutrino magnetic moment in the radiative seesaw model with fermion triplet, Int. J. Mod. Phys. A 30 (2015) 1550007 [arXiv:1202.6394] [INSPIRE].

[46] G.'t Hooft, Naturalness, chiral symmetry, and spontaneous chiral symmetry breaking, NATO Sci. Ser. B 59 (1980) 135 [INSPIRE]. 
[47] J.A. Casas and A. Ibarra, Oscillating neutrinos and $\mu \rightarrow e, \gamma$, Nucl. Phys. B 618 (2001) 171 [hep-ph/0103065] [INSPIRE].

[48] A. Ibarra and G.G. Ross, Neutrino phenomenology: The case of two right-handed neutrinos, Phys. Lett. B 591 (2004) 285 [hep-ph/0312138] [INSPIRE].

[49] N.G. Deshpande and E. Ma, Pattern of Symmetry Breaking with Two Higgs Doublets, Phys. Rev. D 18 (1978) 2574 [INSPIRE].

[50] M.A. Díaz, B. Koch and S. Urrutia-Quiroga, Constraints to Dark Matter from Inert Higgs Doublet Model, arXiv:1511.04429 [INSPIRE].

[51] F.S. Queiroz and C.E. Yaguna, The CTA aims at the Inert Doublet Model, JCAP 02 (2016) 038 [arXiv: 1511.05967] [INSPIRE].

[52] C. Garcia-Cely, M. Gustafsson and A. Ibarra, Probing the Inert Doublet Dark Matter Model with Cherenkov Telescopes, JCAP 02 (2016) 043 [arXiv: 1512.02801] [INSPIRE].

[53] MEG collaboration, J. Adam et al., New constraint on the existence of the $\mu^{+} \rightarrow e^{+} \gamma$ decay, Phys. Rev. Lett. 110 (2013) 201801 [arXiv:1303.0754] [INSPIRE].

[54] SINDRUM collaboration, U. Bellgardt et al., Search for the Decay $\mu^{+} \rightarrow e^{+} e^{+} e^{-}$, Nucl. Phys. B 299 (1988) 1 [INSPIRE].

[55] SINDRUM II collaboration, W.H. Bertl et al., A search for muon to electron conversion in muonic gold, Eur. Phys. J. C 47 (2006) 337 [INSPIRE].

[56] R.H. Bernstein and P.S. Cooper, Charged Lepton Flavor Violation: An Experimenter's Guide, Phys. Rept. 532 (2013) 27 [arXiv:1307.5787] [INSPIRE].

[57] S. Mihara, J.P. Miller, P. Paradisi and G. Piredda, Charged Lepton Flavor-Violation Experiments, Ann. Rev. Nucl. Part. Sci. 63 (2013) 531 [InSPIRE].

[58] G. Signorelli, Charged Lepton Flavor Violation Experiments, arXiv:1307.8346 [INSPIRE].

[59] T. Aushev et al., Physics at Super B Factory, arXiv:1002.5012 [INSPIRE].

[60] Belle, BaBar collaborations, A.J. Bevan et al., The physics of the B Factories, Eur. Phys. J. C 74 (2014) 3026 [arXiv:1406.6311] [InSPIRE].

[61] CMS collaboration, Search for Lepton-Flavour-Violating Decays of the Higgs Boson, Phys. Lett. B 749 (2015) 337 [arXiv:1502.07400] [INSPIRE].

[62] D. Aristizabal Sierra and A. Vicente, Explaining the CMS Higgs flavor violating decay excess, Phys. Rev. D 90 (2014) 115004 [arXiv:1409.7690] [INSPIRE].

[63] I. Doršner, S. Fajfer, A. Greljo, J.F. Kamenik, N. Košnik and I. Nišandžic, New Physics Models Facing Lepton Flavor Violating Higgs Decays at the Percent Level, JHEP 06 (2015) 108 [arXiv: 1502 . 07784] [INSPIRE].

[64] BABAR collaboration, B. Aubert et al., Searches for Lepton Flavor Violation in the Decays $\tau^{ \pm} \rightarrow e^{ \pm} \gamma$ and $\tau^{ \pm} \rightarrow \mu^{ \pm} \gamma$, Phys. Rev. Lett. 104 (2010) 021802 [arXiv:0908.2381] [INSPIRE].

[65] K. Hayasaka et al., Search for Lepton Flavor Violating $\tau$ Decays into Three Leptons with 719 Million Produced $\tau^{+} \tau^{-}$Pairs, Phys. Lett. B 687 (2010) 139 [arXiv:1001.3221] [InSPIRE].

[66] SINDRUM II collaboration, C. Dohmen et al., Test of lepton flavor conservation in $\mu \rightarrow e$ conversion on titanium, Phys. Lett. B 317 (1993) 631 [INSPIRE].

[67] PRIME Working Group collaboration, S. Machida et al., A Letter of Intent to The J-PARC 50-GeV Proton Synchrotron Experiment, http://www-ps.kek.jp/jhf-np/LOIlist/pdf/L25.pdf. 
[68] DeeMe collaboration, H. Natori, DeeMe experiment - An experimental search for a mu-e conversion reaction at J-PARC MLF, Nucl. Phys. Proc. Suppl. 248-250 (2014) 52 [INSPIRE].

[69] W. Porod, F. Staub and A. Vicente, A Flavor Kit for BSM models, Eur. Phys. J. C 74 (2014) 2992 [arXiv: 1405.1434] [INSPIRE].

[70] F. Staub, SARAH, arXiv:0806.0538 [inSPIRE].

[71] F. Staub, From Superpotential to Model Files for FeynArts and CalcHep/CompHEP, Comput. Phys. Commun. 181 (2010) 1077 [arXiv:0909.2863] [INSPIRE].

[72] F. Staub, Automatic Calculation of supersymmetric Renormalization Group Equations and Self Energies, Comput. Phys. Commun. 182 (2011) 808 [arXiv: 1002.0840] [INSPIRE].

[73] F. Staub, SARAH 3.2: Dirac Gauginos, UFO output and more, Comput. Phys. Commun. 184 (2013) 1792 [arXiv: 1207.0906] [INSPIRE].

[74] F. Staub, SARAH 4: A tool for (not only SUSY) model builders, Comput. Phys. Commun. 185 (2014) 1773 [arXiv: 1309.7223] [INSPIRE].

[75] W. Porod, SPheno, a program for calculating supersymmetric spectra, SUSY particle decays and SUSY particle production at $e^{+} e^{-}$colliders, Comput. Phys. Commun. 153 (2003) 275 [hep-ph/0301101] [INSPIRE].

[76] W. Porod and F. Staub, SPheno 3.1: Extensions including flavour, CP-phases and models beyond the MSSM, Comput. Phys. Commun. 183 (2012) 2458 [arXiv:1104.1573] [INSPIRE].

[77] D.V. Forero, M. Tortola and J.W.F. Valle, Neutrino oscillations refitted, Phys. Rev. D 90 (2014) 093006 [arXiv: 1405.7540] [INSPIRE].

[78] A. Merle, M. Platscher, N. Rojas, J.W.F. Valle and A. Vicente, Consistency of WIMP Dark Matter as radiative neutrino mass messenger, JHEP 07 (2016) 013 [arXiv:1603.05685] [INSPIRE].

[79] F. von der Pahlen, G. Palacio, D. Restrepo and O. Zapata, Radiative Type III Seesaw Model and its collider phenomenology, arXiv:1605.01129 [INSPIRE].

[80] J. Hisano, T. Moroi, K. Tobe and M. Yamaguchi, Lepton flavor violation via right-handed neutrino Yukawa couplings in supersymmetric standard model, Phys. Rev. D 53 (1996) 2442 [hep-ph/9510309] [INSPIRE].

[81] T.S. Kosmas, S. Kovalenko and I. Schmidt, Nuclear $\mu^{-}-e^{-}$conversion in strange quark sea, Phys. Lett. B 511 (2001) 203 [hep-ph/0102101] [INSPIRE].

[82] A. Crivellin, M. Hoferichter and M. Procura, Improved predictions for $\mu \rightarrow e$ conversion in nuclei and Higgs-induced lepton flavor violation, Phys. Rev. D 89 (2014) 093024 [arXiv: 1404.7134] [INSPIRE].

[83] Y. Kuno and Y. Okada, Muon decay and physics beyond the standard model, Rev. Mod. Phys. 73 (2001) 151 [hep-ph/9909265] [INSPIRE].

[84] H.C. Chiang, E. Oset, T.S. Kosmas, A. Faessler and J.D. Vergados, Coherent and incoherent $\left(\mu^{-}, e^{-}\right)$conversion in nuclei, Nucl. Phys. A 559 (1993) 526 [INSPIRE]. 